\title{
Correction to: Intrinsic curvature of curves and surfaces and a Gauss-Bonnet theorem in the Heisenberg group
}

\section{Zoltán M. Balogh ${ }^{1}$. Jeremy T. Tyson ${ }^{2}$. Eugenio Vecchi ${ }^{3}$}

Published online: 14 February 2019

○ Springer-Verlag GmbH Germany, part of Springer Nature 2019

\section{Correction to: Math. Z. (2017) 287:1-38 https://doi.org/10.1007/s00209-016-1815-6}

In the publication [1] there is an unfortunate computational error, which however does not affect the correctness of the main results.

Let us recall some notation from the paper. By $\gamma:[a, b] \rightarrow \mathbb{R}^{3}$ we denote a $\mathcal{C}^{2}$ smooth parametrized regular curve $t \rightarrow \gamma(t)=\left(\gamma_{1}(t), \gamma_{2}(t), \gamma_{3}(t)\right)$. The action of the standard contact form $\omega=d x_{3}-\frac{1}{2}\left(x_{1} d x_{2}-x_{2} d x_{1}\right)$ on $\gamma$ is denoted by

$$
\omega(\dot{\gamma})=\omega(\dot{\gamma})(t)=\dot{\gamma}_{3}(t)-\frac{1}{2}\left(\gamma_{1}(t) \dot{\gamma}_{2}(t)-\gamma_{2}(t) \dot{\gamma}_{1}(t)\right)
$$

A point $t_{0} \in[a, b]$ is called horizontal if and only if $\omega(\dot{\gamma})\left(t_{0}\right)=0$. The mistake in the paper arises due to a statement implicitly assumed in the proof of Lemma 3.4, that an an horizontal point we also have that $\omega(\ddot{\gamma})\left(t_{0}\right)=0$, where

$$
\omega(\ddot{\gamma})=\omega(\ddot{\gamma})(t)=\ddot{\gamma}_{3}(t)-\frac{1}{2}\left(\gamma_{1}(t) \ddot{\gamma}_{2}(t)-\gamma_{2}(t) \ddot{\gamma}_{1}(t)\right) .
$$

ZMB and EV were supported by the Swiss National Science Foundation Grant No. 200020-146477, and have also received funding from the People Programme (Marie Curie Actions) of the European Union's Seventh Framework Programme FP7/2007-2013/ under REA grant agreement No. 607643 (Grant MaNET 'Metric Analysis for Emergent Technologies'). JTT acknowledges support from U.S. National Science Foundation Grants DMS-1201875 and DMS-1600650 and Simons Foundation Collaboration Grant 353627.

The original article can be found online at https://doi.org/10.1007/s00209-016-1815-6.

\section{Jeremy T. Tyson}

tyson@illinois.edu

Zoltán M. Balogh

zoltan.balogh@math.unibe.ch

\section{Eugenio Vecchi}

vecchi@mat.uniroma1.it

1 Mathematisches Institut, Universität Bern, Sidlerstrasse 5, 3012 Bern, Switzerland

2 Department of Mathematics, University of Illinois, 1409 West Green St., Urbana, IL 61801, USA

3 Dipartimento di Matematica, Università di Bologna, Piazza di Porta San Donato 5, 40126 Bologna, Italy 
This fact is in general not true. As a result, various statements in the paper, including the second formula in equation (1.1), equation (3.4), the second part of equation (3.10), and the second displayed equations in both Lemma 4.8 and Proposition 4.13, do not hold for all horizontal points.

However, noticing that $\omega(\ddot{\gamma})=\frac{d}{d t} \omega(\dot{\gamma})$ we see that the assertion $\omega(\ddot{\gamma})\left(t_{0}\right)=0$ is still true for horizontal points that arise as accumulation points of other horizontal points. Since the parameterizing interval is compact, there are at most a finite number of isolated horizontal points $t_{1}, \ldots, t_{N}$ at which the quantity $\omega(\ddot{\gamma})\left(t_{i}\right)$ may be nonzero, and hence all of the preceding formulas hold at all points of $[a, b]$ except for this finite number of isolated points.

The main result of the paper, Theorem 1.1, is not affected by these corrections since its proof is based on an approximation argument relying on the Lebesgue dominated convergence theorem. In the application of this theorem a set of countably many points can be ignored as a null set, and the proof works as indicated in Section 6 of the paper.

Acknowledgements We are grateful to Derek Jung and Maxim Tryamkin for pointing out the error in the proof of Lemma 3.4.

\section{Reference}

1. Balogh, Z.M., Tyson, J.T., Vecchi, E.: Intrinsic curvature of curves and surfaces and a Gauss-Bonnet theorem in the Heisenberg group. Math. Z. 287, 1-38 (2017)

Publisher's Note Springer Nature remains neutral with regard to jurisdictional claims in published maps and institutional affiliations. 